\title{
Transition Metal-doped Nickel Phosphide Nanoparticles as Electro- and Photocatalysts for Hydrogen Generation Reactions
}

\author{
Ho-Wing Man ${ }^{1}$, Chui-Shan Tsang ${ }^{1}$, Molly Meng-Jung Li ${ }^{2}$, Jiaying Mo ${ }^{2}$, Bolong Huang ${ }^{1}$, \\ Lawrence Yoon Suk Lee ${ }^{l *}$, Yun-chung Leung ${ }^{l}$, Kwok-Yin Wong ${ }^{l}$, and Shik Chi Edman \\ Tsang $^{1,2 *}$
}

${ }^{1}$ Department of Applied Biology and Chemical Technology and the State Key Laboratory of Chemical Biology and Drug Discovery, The Hong Kong Polytechnic University, Hung Hom, Kowloon, Hong Kong

${ }^{2}$ Department of Chemistry, University of Oxford, Oxford, OX1 3QR, UK

*Corresponding e-mails: edman.tsang@chem.ox.ac.uk and lawrence.ys.lee@polyu.edu.hk

\begin{abstract}
Transition metal-doped nickel phosphide nanoparticles with metallic properties are prepared by a simple and facile wet-chemical method. It is shown for the first time that these transition metals: iron, cobalt, manganese, and molybdenum, can atomically substitute nickel in the parent hcp phosphide lattice as a single phase without significant change in its metallic structure and morphology. They are employed as electro- and photocatalysts for hydrogen evolution reaction, which show highly tunable activities dependent on electron filling of their metallic bands and $\mathrm{H}$ coverage according to our experimental and theoretical rationalizations. Molybdenum-doped nickel phosphide nanoparticle with lower $\mathrm{H}$ coverage exhibits the best hydrogen evolution performance in electrocatalytic hydrogen evolution reaction, which also shows excellent photocatalytic hydrogen production with organic photosensitizer. In addition, cobalt-doped nickel phosphide nanoparticle with higher $\mathrm{H}$ coverage with aqueous photosensitizer gives more superior hydrogen evolution rate.
\end{abstract}

Keywords: metal doping, nickel phosphide, electrocatalysis, photocatalysis, hydrogen evolution reaction 
With the continuous and exponential increase in global energy demand and severe environmental issues, sustainable and renewable energy sources are required to replace fossil fuels. Hydrogen fuel is one of the good candidates because of its environmental friendly nature and high energy output through combustion.[1-3] One of the most promising ways to produce hydrogen is through hydrogen evolution reaction (HER), by either electro- or photocatalysis. In electrocatalytic HER, platinum group metals (PGMs), such as platinum, are found to be the most active HER electrocatalysts.[4] In addition to electrocatalytic HER, PGMs are also employed in photoelectrochemical HER.[5] However, PGMs are scarce and expensive to be used. Alternatives such as $\mathrm{MoS}_{2}$ and $\mathrm{TiO}_{2}$, composed of cheaper and more earth-abundant elements, show promising catalytic activities in either electro- or photocatalytic HER.[6,7]

Recently, transition metal phosphides (TMPs) with comparable metallic properties to carry out reversible dissociation or association of $\mathrm{H}$ to $\mathrm{H}_{2}$ have drawn much attention as potential HER catalysts. Particularly for nickel phosphide, Liu and Rodriguez have conducted DFT calculations on estimating the Gibbs free energy of hydrogen adsorption $\left(\Delta G_{\mathrm{H}}\right)$, a descriptor for HER, on $\mathrm{Ni}_{2} \mathrm{P}(001)$ surface.[8] The calculation suggested that $\mathrm{Ni}_{2} \mathrm{P}(001)$ surface could even show a higher activity under some specific conditions than $\mathrm{Pt}$ on a thermodynamic basis. After that, $\mathrm{Ni}_{2} \mathrm{P}$ nanoparticle was experimentally demonstrated to display excellent electrocatalytic HER activity under acidic conditions.[9] Further improvements were made on nickel phosphide through the preparation of different crystal phases[10-12] and the incorporation of carbonaceous materials.[13-15] Most recently, it has been also demonstrated that nickel phosphide can be used as an active component in HER photocatalysis, when it is mixed with semiconductor phases, including $\mathrm{CdS}$ and $\mathrm{g}-\mathrm{C}_{3} \mathrm{~N}_{4}$.[1618] Electrons are thought to be transferred from the photo-excited conduction bands of semiconductors to $\mathrm{Ni}_{2} \mathrm{P}$, which eventually lead to hydrogen production from the phosphide surface. In order to get an optimal $\mathrm{H}_{2}$ production rate, tuning appropriate metal- $\mathrm{H}$ bond strength is important to facilitate $\mathrm{H}^{+}$adsorption and reduction (Volmer step) and surface combination and desorption of dihydrogen (Tafel step). $[19,20]$ The incorporation of another metal surface of different metal-H bond strength to form a composite is a common strategy, which speeds up both Vomer and Tafel steps. This approach has been widely studied on metals and metal oxide composites in both theoretical and experimental aspects.[21-24] Attempts have been made on nickel phosphide-based systems where other transition metals are incorporated with the nickel phosphide as separated phases in the composites.[25-27] In particular, nickel and cobalt phosphides are prepared with $\mathrm{g}-\mathrm{C}_{3} \mathrm{~N}_{4}$ to form a composite and employed as a HER photocatalyst, achieving an excellent hydrogen production rate.[28] Herein, we report that transition metals, including iron, cobalt, manganese, and molybdenum, are successfully incorporated into nickel phosphide crystal lattice as a single phase to form transition metal-doped nickel phosphide nanoparticles through simple and facile wet-chemical method. The as-prepared transition metal-doped nickel phosphide (NiMP) nanoparticles are shown to have improvements both in electro-and photocatalytic HER over pristine nickel phosphide $\left(\mathrm{Ni}_{2} \mathrm{P}\right)$ nanoparticle.

\section{Experimental Section}


Synthesis of pristine and metal-doped nickel phosphide nanoparticles.

Typically, $1 \mathrm{mmol}$ of nickel(II) acetate tetrahydrate was mixed in $5 \mathrm{~mL}$ of oleylamine in a 100 $\mathrm{mL}$ three-necked round-bottomed flask. The mixture was put under vacuum to remove adventitious oxygen and moisture. $11.2 \mathrm{mmol}$ of trioctylphosphine (TOP) and equimolar of trioctylphosphine oxide (TOPO) were added to the flask sequentially. The reaction mixture was heated at $300{ }^{\circ} \mathrm{C}$ for $2 \mathrm{~h}$ under $\mathrm{N}_{2}$ protection. After $2 \mathrm{~h}$ reaction, the reaction mixture was cooled down naturally by removal of heat source. $30 \mathrm{~mL}$ absolute ethanol was added, and precipitate was collected by centrifugation at $9000 \mathrm{rpm}$ for $5 \mathrm{~min}$. Supernatant was discarded and precipitate was purified by redispersion/precipitation process using chloroform and ethanol. The process was repeated for three times and purified product was dried and kept under vacuum. The synthetic procedures of metal-doped nickel phosphide nanoparticles were similar to the pristine one. $0.5 \mathrm{mmol}$ of iron(II) acetate, manganese(III) acetate dihydrate, and cobalt(II) acetate were added with $0.5 \mathrm{mmol}$ nickel(II) acetate tetrahydrate for the preparation of iron-, manganese-, and cobalt-doped nickel phosphide nanoparticles. $0.25 \mathrm{mmol}$ of molybdenum acetate dimer was used to maintain metal ion concentration for preparing molybdenum-doped nickel phosphide nanoparticle.

\section{Characterization.}

X-Ray powder diffraction pattern was obtained using Rigaku SmartLab with scan rate of $1^{\circ}$ $\min ^{-1}$. Powder sample was placed on a silicon wafer and pressed using a glass slide. Diffraction pattern from $2 \theta=35^{\circ}$ to $60^{\circ}$ was obtained for pristine and various metal-doped nickel phosphide nanoparticles. As-prepared powder samples were dispersed and sonicated in chloroform to obtain well-dispersed suspension. 20-40 $\mu \mathrm{L}$ of suspension was added on a 400mesh copper grid with holely carbon coating and dried naturally. Images from transmission electron microscopy were obtained using a scanning transmission electron microscope (JOEL JEM-2100F). Selected area electron diffraction (SAED) and energy-dispersive X-ray spectroscopy (EDX) were carried out in tandem in transmission electron microscopy. X-Ray photoelectron spectra of pristine and various metal-doped nickel phosphide nanoparticles were obtained using X-Ray photoelectron spectrophotometer (ESCALAB $250 \mathrm{Xi}$ ) with approximately $20 \mathrm{mg}$ of powder sample. Calibration was done using binding energy of $\mathrm{C} 1 \mathrm{~s}$ energy level at $284.8 \mathrm{eV}$ as reference. Local structures surrounding $\mathrm{Ni}$ and $\mathrm{Fe}$ atoms were probed by using extended X-ray absorption fine structure (EXAFS) technique in B18 Diamond light source. A Si(111) Double Crystal Monochromator (DCM) was used to scan the photon energy. The energy resolution $(\Delta \mathrm{E} / \mathrm{E})$ for the incident X-ray photons was estimated to be $2 \times 10^{-4}$. Transmission mode was adopted for Ni K-edge and Fe K-edge EXAFS measurements. To ascertain the reproducibility of experimental data, at least two scan sets were collected and compared for each sample. The EXAFS data analysis was performed using IFEFFIT 1 with Horae packages 2 (Athena and Artemes). The spectra were calibrated with Ni and Fe metal foils as the references to avoid energy shifts of the samples. The amplitude reduction factors were obtained from EXAFS data analysis of the references, which were used as the fixed input parameters in the data fitting to allow the refinement in the coordination number of the absorption element. In this work, the first shell data analyses under the assumption of single scattering were performed with the errors estimated by R-factor. 


\section{Electrochemical Measurement.}

To prepare the working electrode, catalyst ink was prepared by dissolving $2.5 \mathrm{mg}$ metaldoped nickel phosphide nanoparticles in a solution containing $460 \mu \mathrm{L}$ ethanol-water mixture (3:7) and $40 \mu \mathrm{L} 5$ wt.\% Nafion solution. Working electrode was prepared by drop-casting 10 $\mu \mathrm{L}$ of catalyst ink on a glassy carbon electrode (GCE, $\mathrm{d}=3 \mathrm{~mm}$, surface area $=0.07 \mathrm{~cm}^{2}$ ). The catalyst ink was dried naturally. Electrochemical measurements were performed using a CHI potentiostat (CHI1030A). Electrocatalytic hydrogen evolution reaction was conducted in a typical three-electrode system, using Pt mesh and saturated calomel electrode (SCE) as the counter and reference electrode, respectively, in $1 \mathrm{M}$ potassium hydroxide solution $(\mathrm{pH}=13)$. The electrolyte solution was purged with Ar for $30 \mathrm{~min}$ before measurements to get rid of dissolved oxygen and provide inert atmosphere. Linear sweep voltammetry (LSV) was carried out at room temperature in a potential range from -1 to $-1.9 \mathrm{~V}$ with a scan rate of $5 \mathrm{mV} \mathrm{s}^{-1}$. The potential measurements against saturated calomel electrode (SCE) were converted with respect to the reversible hydrogen electrode (RHE) according to the following equation [29]:

$$
\mathrm{E}_{\mathrm{RHE}}=\mathrm{E}_{\mathrm{SCE}}+0.244+0.0592(\mathrm{pH})
$$

\section{Photocatalytic Testing}

$5 \mathrm{mg}$ of catalyst and $64 \mathrm{mg}$ of dye were mixed in a $55 \mathrm{~mL}$ quartz flask containing $20 \mathrm{~mL}$ TEOA-DMF $(15 \% \mathrm{v} / \mathrm{v})$ solution. The solution was sonicated for $15 \mathrm{~min}$ to achieve a good dispersion and degassed with Ar for 15 min afterwards. The flask was illuminated with a 450 W Xe lamp equipped with an AM1.5 filter (Newport OPSA1000) for $2 \mathrm{~h}$. Gas generated in the headspace $(250 \mathrm{~mL})$ was withdrawn using an air-tight syringe and analyzed using a gas chromatograph equipped with thermal conductivity detector (GC-TCD, Agilent 7890B) and ValcoPLOT Molesieve $5 \AA$ PLOT column (CFS-X3053-500). The amount of $\mathrm{H}_{2}$ generated was calculated based on the calibration curve. $\mathrm{H}_{2}$ production rate was calculated based on the metal content of the catalyst determined by energy dispersive X-ray spectroscopy.

\section{Photostability test}

The stability against photo-corrosion was tested out using NiMoP nanoparticle. The preparation of solution and analysis of hydrogen produced by photocatalysis are the same as the previous descriptions. The duration of one photocatalysis run was set to $5 \mathrm{~h}$ and five consecutive runs were conducted. After each run, NiMoP nanoparticle was collected by centrifugation and re-suspended in a fresh DMF solution with $\left[\mathrm{Ru}(\mathrm{bpy})_{3}\right]\left(\mathrm{ClO}_{4}\right)_{2}$ and triethanolamine.

\section{Results and Discussion}

To synthesize transition metal-doped nickel phosphide (NiMP) nanoparticles, $\mathrm{Ni}(\mathrm{OAc})_{2} \cdot 4 \mathrm{H}_{2} \mathrm{O}$ and $\mathrm{M}(\mathrm{OAc})_{\mathrm{x}}$ were heated in the presence of oleylamine, trioctylphosphine (TOP), and trioctylphosphine oxide (TOPO) at $300{ }^{\circ} \mathrm{C}$ for $2 \mathrm{~h}$. Figure 1 shows the X-ray diffraction (XRD) patterns of pristine nickel phosphide $\left(\mathrm{Ni}_{2} \mathrm{P}\right)$ and all NiMP nanoparticles which are in good match to the reported $\mathrm{Ni}_{2} \mathrm{P}$ crystal structure (PDF\#-65-1989).

TEM images show the formation of all spherical nanoparticles with an average diameter of approximately $7 \mathrm{~nm}$, except for NiMoP nanoparticles of having $5.3 \pm 1 \mathrm{~nm}$ (Figure 2). 
Electron diffraction patterns of all samples tested are almost identical, consisting four rings of diffraction. The predominant (111) lattice fringe shows a $d$-spacing of $0.22 \mathrm{~nm}$. The selected area electron diffraction (SAED) pattern shows the rings of diffraction, where the innermost ring is indexed as (111) lattice plane and the outermost ring corresponds to (300) lattice plane. The measurements of $d$-spacing and electron diffraction pattern are in good agreements with XRD patterns mentioned previously. The elemental composition is determined by EDX (Figures S1-S5, Table S1 in Supplementary Information). In particular, the normalized ratio of elements in $\mathrm{NiFeP}$ nanoparticle (nickel-to-iron-to-phosphorus) is 1:0.1:0.7. An overall 6\% of iron is successfully doped into the nickel phosphide crystal lattice.

X-ray photoelectron spectroscopy (XPS) shows that the as-synthesized NiMP nanoparticles display metallic properties where the binding energies of different metalphosphorus bonds are similar to metals at Fermi state. XPS spectra and detailed peak deconvolutions are shown in Supplementary Information (Figures S6-S10). In addition to the above characterization, typical extended X-ray absorption fine structure (EXAFS) measurement was carried out on $\mathrm{Ni}_{2} \mathrm{P}$ and $\mathrm{NiFeP}$ nanoparticles to investigate the local bonding environments of metals (Table 1 and Figures S11-S13). Ni-P and Ni-Ni bonds in $\mathrm{NiFeP}$ nanoparticle are determined as 2.24 and $2.59 \AA$, respectively, which match with those in $\mathrm{Ni}_{2} \mathrm{P}$ nanoparticle in $h c p$ structure as shown in SI $(2.25$ and $2.59 \AA$ for Ni-P and Ni-Ni bonds, respectively). Meanwhile, the fitted Fe-Ni and Fe-P bonds are very similar to Ni-P and $\mathrm{Ni}-\mathrm{Ni}$ as 2.63 and $2.21 \AA$, respectively. The bond lengths in iron-doped and pristine nickel phosphide nanoparticles are comparable which shows the incorporation of iron in nickel phosphide crystal lattice does not induce significant changes in bond strengths of existing $\mathrm{Ni}-\mathrm{Ni}$ and Ni-P bonds. Typical coordination numbers in Ni-Ni and Ni-P for $\mathrm{Ni}_{2} \mathrm{P}$ are 3 for both, which are close to our fittings as shown in Table 1, having coordination numbers for $\mathrm{Ni}-\mathrm{Ni}$ and Ni-P as 3.3 and 2.9, respectively.[30] There is, however, a decrease in the coordination numbers when $\mathrm{Fe}$ is introduced into the crystal lattice. The coordination numbers for Ni-Ni and Ni-P in NiFeP nanoparticles are 2.5 and 2.3, respectively, whereas those for $\mathrm{Fe}-\mathrm{Ni}$ and Fe-P are determined to be 3.6 and 3.7, respectively. The decreased coordination numbers of $\mathrm{Ni}-\mathrm{Ni}$ and $\mathrm{Ni}-\mathrm{P}$ indicate the successful substitution of $\mathrm{Ni}$ with $\mathrm{Fe}$. In addition, the change in coordination number does not reveal a perfect substitution since there is a degree of mismatch between the added coordination numbers of Ni-P and Fe-P in NiFeP nanoparticle and that of Ni-P in $\mathrm{Ni}_{2} \mathrm{P}$ nanoparticle. But, the basic $h c p$ structure is clearly maintained. From the above control experiments for $\mathrm{NiFeP}$ nanoparticle synthesis, it was confirmed that iron present in the reaction environment can be incorporated into nickel phosphide crystal lattice without making much significant effect on the morphology, crystal structure, and bonding environment of the resulting nanoparticles despite the large degree of Fe incorporation.

\section{Electro- and photocatalytic performances}

Working electrodes of our samples were prepared by drop-casting the corresponding catalyst ink on glassy carbon electrodes, with a loading of $0.7 \mathrm{mg} \mathrm{cm}{ }^{-2}$. Linear sweep voltammograms were obtained from 0 to $-0.8 \mathrm{~V}$ ( $v s$. RHE, unless stated otherwise) in $1 \mathrm{M}$ potassium hydroxide solution (Figure 3a). The overpotential of $\mathrm{Ni}_{2} \mathrm{P}$ is determined as $0.71 \mathrm{~V}$ to reach a low current density of $10 \mathrm{~mA} \mathrm{~cm}$-2 (low H-coverage). For all NiMP nanoparticles prepared, cathodic shifts are seen on all the corresponding polarization plots, indicating that 
enhancement in HER catalytic performance. The overpotential at $10 \mathrm{~mA} \mathrm{~cm}{ }^{-2}$ for $\mathrm{Mo}, \mathrm{Mn}, \mathrm{Fe}$, Co-doped, and pristine nickel phosphide (abbreviated as NiMoP, NiMnP, NiFeP, NiCoP, $\mathrm{Ni}_{2} \mathrm{P}$ ) nanoparticles are $0.4,0.49,0.69,0.53$, and $0.71 \mathrm{~V}$, respectively. This order almost inversely follows their number of electrons in $d$ orbital, $\left(\operatorname{Mo}\left(d^{6}\right) ; \operatorname{Mn}\left(d^{7}\right) ; \operatorname{Fe}\left(d^{8}\right) ; \operatorname{Co}\left(d^{9}\right)\right.$ and $\left.\mathrm{Ni}\left(d^{10}\right)\right)$, with exception of the relative positions of $\mathrm{Fe}$ and Co. This reflects the $d^{8}$ electron contribution of $\mathrm{Ni}$ to the metallic bands is perhaps too much above the optimal chemisorption of $\mathrm{H}^{+}$and reduction to lead to lower HER activity. Substitution of Ni with elements of lower number of $d$-electrons at 1:1 of Ni:M ratio appears to shift towards the optimal value for the HER.[31,32] However, the precise order of metal dopers is dependent on the $\mathrm{H}$ coverage (where their current density values are compared) in some cases, especially for Co which is highly sensitive to the coverage and may switch with Fe in their positions, as shown later (Figure 9). In addition, all the samples tested yielded relatively high Tafel slopes shown in Figure 3b $\left(>100 \mathrm{mV} \mathrm{dec}^{-1}\right)$. These slopes indicate the rate determining step is associated with Volmer step where the adsorption of hydrogen on catalyst surface at low H coverage is involved. The result is consistent with the DFT calculations by Liu and Rodriguez[8] in which the hydrophobic $\mathrm{Ni}_{2} \mathrm{P}$ nanoparticle has a positive value of Gibbs free energy of hydrogen adsorption $\left(\Delta G_{\mathrm{H}}=0.31 \mathrm{eV}\right)$. The exchange current densities of $\mathrm{Ni}_{2} \mathrm{P}$ and various NiMP nanoparticles are tabulated in Table 2. NiMoP nanoparticle shows the lowest overpotential and one of the highest exchange current densities (high degree of mobile electrons), giving the best overall HER activity among the samples tested. Doping of Mo enhances the HER catalytic property from delocalization of $4 d$-electrons.[32,33] Mo, the most $d$-electron deficient transition metal among samples tested, as well as with the more outstretching nature of $4 d$ compared to $3 d$ orbital, leads to the largest degree of $d$-electron delocalization. However, compared to $\mathrm{Pt}$, the best benchmark hydrogen-evolving electrocatalyst (a Tafel slope of 61 $\mathrm{mV} \operatorname{dec}^{-1}$ ), NiMoP nanoparticle shows moderate electrocatalytic activity in alkaline medium.

The positions of valence bands of different metallic NiMP nanoparticles were determined by valence band-X ray photoelectron spectroscopy (VB-XPS) and are shown in Figure 4. Typical nature of metallic bands of these structures between 0 to $-10 \mathrm{eV}$ with the maxima value lower than the thermodynamic proton reduction potential $(0.00 \mathrm{eV})$ indicate that activation energy must be required for the hydrogen production.

Apart from using electromotive force as excitation source, NiMP nanoparticle is also employed for HER by photoexcitation. Photocatalytic HER was carried out in the presence of photosensitizer, tris(2,2'-bipyridyl)ruthenium(II) perchlorate $\left(\mathrm{Ru}(\mathrm{bpy})_{3}\left(\mathrm{ClO}_{4}\right)_{2}\right)$, which has a strong characteristic absorption in visible region $(455 \mathrm{~nm})$ with a high extinction coefficient.[34] Upon illumination, photo-generated electrons from this photosensitizer could be injected into the metallic bands of NiMP for the proton reduction. The rapid electron transfer could also reduce the re-combination of photo-generated electrons and holes from the sensitizer to heat.[35] In addition to photosensitizer, triethanolamine was added as a sacrificial agent, which assists the regeneration of photosensitizer to ground state. The irradiation of $\mathrm{NiFeP}$ nanoparticles in the presence of $\left[\mathrm{Ru}(\mathrm{bpy})_{3}\right]\left(\mathrm{ClO}_{4}\right)_{2}$ in $\mathrm{N}, \mathrm{N}^{\prime}$-dimethylformamide (DMF) using $450 \mathrm{~W}$ solar simulator with air mass 1.5 filter for one hour resulted in the formation of $486 \mu \mathrm{mol}$ of $\mathrm{H}_{2}$. As far as we are aware, this photocatalytic HER activity is amongst the best reported in the literature (Table S3). In the two control experiments conducted under the same experimental conditions, only small amount of $\mathrm{H}_{2}(24 \mu \mathrm{mol})$ was detected with the presence 
of $\left[\mathrm{Ru}(\mathrm{bpy})_{3}\right]\left(\mathrm{ClO}_{4}\right)_{2}$ only, while no $\mathrm{H}_{2}$ was detected with $\mathrm{NiFeP}$ nanoparticle only (Figure 5a). These results confirm that NiFeP nanoparticle alone cannot carry out the photocatalytic HER, but they can catalyze the HER with excellent reactivity with the assistance of a light absorber, $\left[\mathrm{Ru}(\mathrm{bpy})_{3}\right]\left(\mathrm{ClO}_{4}\right)_{2}$. It demonstrates that the excitons generated in $\left[\mathrm{Ru}(\mathrm{bpy})_{3}\right]\left(\mathrm{ClO}_{4}\right)_{2}$ are effectively transferred to NiFeP nanoparticle, where they are used to catalyze the HER.

After assuring the synergetic effect between ruthenium complex and NiFeP nanoparticle, the reactivities of other NiMP nanoparticles were also studied (Figure 5b). In general, metal doping in nickel phosphide nanoparticles shows a positive effect on the performance in dyesensitized photocatalytic HER. The $\mathrm{Ni}_{2} \mathrm{P}$ nanoparticle produces $\mathrm{H}_{2}$ at a rate of $174 \mathrm{mmol} \mathrm{h}^{-}$ ${ }^{1} \mathrm{~g}^{-1}$, while $\mathrm{NiFeP}, \mathrm{NiCoP}, \mathrm{NiMnP}$, and NiMoP all show a higher hydrogen production rate of $189,210,259$, and $268 \mathrm{mmol} \mathrm{h}^{-1} \mathrm{~g}^{-1}$, respectively. The rank of photocatalytic activity is as follows: $\mathrm{Ni}_{2} \mathrm{P}<\mathrm{NiFeP}<\mathrm{NiCoP}<\mathrm{NiMnP}<\mathrm{NiMoP}$.

Notice that the order of photocatalytic activity of different NiMP nanoparticles is identical to the decreasing order of overpotential for electrochemical hydrogen evolution. Such identical trend in HER catalytic activity is attributed to the same nature of active site on metal catalyst surface for $\mathrm{H}_{2}$ production. Clearly, NiMP nanoparticles in dye-sensitized photocatalytic system act as the reaction center for hydrogen production upon receiving electrons from the excited dye. In order to further investigate the effect of metal doping on photocatalytic HER rate, the nickel-to-cobalt precursor ratio has been systematically varied from 3:1 to 1:2 during the synthesis of NiCoP nanoparticles. XRD, TEM images, elemental composition of NiCoP nanoparticles prepared under different nickel-to-cobalt ratio are shown in Supplementary Information (Figures 6, S14, and Table S2). Their corresponding hydrogen production rates were measured and are shown in Figure 7. A volcano-type activity relationship with respect to the Ni:Co precursor ratio can be seen, where the optimal value lies on the 1:1 ratio (the highest $\mathrm{H}_{2}$ production rate of $209 \mathrm{mmol} \mathrm{h}^{-1} \mathrm{~g}^{-1}$ ). Lowering cobalt content leads to a decrease in $\mathrm{H}_{2}$ producing ability. As seen, Ni:Co ratios of 2:1 and 3:1 (abbreviated as $\mathrm{NiCoP} 21$ and $\mathrm{NiCoP} 31)$ show the $\mathrm{H}_{2}$ production rate of 180 and $147 \mathrm{mmol} \mathrm{h}^{-1} \mathrm{~g}^{-1}$, respectively. On the other hand, increasing the Co contents beyond the optimal value to 1:2 Ni:Co ratio (NiCoP12) give a $\mathrm{H}_{2}$ production rate of $140 \mathrm{mmol} \mathrm{h}^{-1} \mathrm{~g}^{-1}$. It is thought that $\mathrm{H}_{2}$ production rate is related to the delicate degree of electron filling of metallic bands as discussed with keeping the same NiMP hcp structure. Apparently, NiCoP11 with 1:1 Co substitution to Ni shows the optimal $d$-electron filling for the highest $\mathrm{H}_{2}$ production rate.

Stability test was carried out with NiMoP nanoparticle in $\mathrm{Ru}(\mathrm{bpy}) 3^{2+}$-sensitized photocatalytic system (Figure 8). The test was done in five separate runs of $5 \mathrm{~h}$ for the total duration of $25 \mathrm{~h}$. After each run, NiMoP nanoparticle was isolated and photosensitizer and sacrificial agent were recharged. NiMoP nanoparticle clearly shows a good stability during the photocatalytic HER. The total photogenerated hydrogen quantities from the first and fifth runs are within $80 \%$ over $25 \mathrm{~h}$ despite a small loss in sample during the retests. Also, we confirmed that the morphology, crystal structure, and composition of NiMoP nanoparticle are retained without significant change after the photocatalysis from the post-HER characterizations (Figure S15).

Due to poor solubility of $\mathrm{Ru}(\mathrm{bpy}) 3_{3}{ }^{2+}$ photosensitizer in water, the photocatalytic HER activities of NiMP nanoparticles were acquired in DMF. Attempts to collect HER activities in water were also carried out using water-soluble eosin $\mathrm{Y}$ photosensitizer, the dye also with a 
characteristic absorption in visible region (Figure S16). The conjugate base of eosin $\mathrm{Y}$ renders its high solubility in water. The NiMP nanoparticles were tested as HER photocatalysts in water and the results are summarized in Figure 9. The HER activities between samples do not seem to deviate much, ranging from $15.3 \mathrm{mmol} \mathrm{h}^{-1} \mathrm{~g}^{-1}$ for NiMoP nanoparticle, $16.7 \mathrm{mmol} \mathrm{h}^{-1} \mathrm{~g}^{-1}$ for NiMnP nanoparticle, $17 \mathrm{mmol} \mathrm{h}^{-1} \mathrm{~g}^{-1}$ for $\mathrm{NiFeP}$ nanoparticle, $17.7 \mathrm{mmol} \mathrm{h}^{-1} \mathrm{~g}^{-1}$ for $\mathrm{Ni}_{2} \mathrm{P}$ nanoparticle, to $20.4 \mathrm{mmol} \mathrm{h}^{-1} \mathrm{~g}^{-1}$ for $\mathrm{NiCoP}$ nanoparticle. The low activities may reflect the poor electron transfer of eosin $\mathrm{Y}$ compared to the $\mathrm{Ru}$ complex but this system offers one of the highest reported photocatalytic HER activities in water compared to literature (Table S4). It is however, interesting to note that this order of catalytic activity in the presence of eosin $\mathrm{Y}$ is very different from that of $\left[\mathrm{Ru}(\text { bpy })_{3}\right]\left(\mathrm{ClO}_{4}\right)_{2}$. We attribute the main difference to the proton concentration that is much higher in water than in DMF. This will affect critically on the degree of hydrogen coverage on their surfaces. It has been shown that increasing hydrogen coverage can increase the Gibbs free energy of hydrogen adsorption $\left(\Delta \mathrm{G}_{\mathrm{H}}\right)$. [36,37]

To verify this point, a free energy $(\Delta G)$ calculation on the HER on the NiP structure was first modelled (Figure S17) and the result is shown in Figure 10 with different $\mathrm{H}$ coverage values. In these $\Delta G$ calculations, the total energy calculation was carried out by DFT method and the $\mathrm{H}$ atom was placed on the Ni site of the pristine NiP surface. Figure 10a shows that the dramatic $\mathrm{H}$ coverage effect on HER efficiency of the pristine NiP surface lattice. By comparing 1/8 monolayer (ML) to $1 / 4 \mathrm{ML}$ of $\mathrm{H}$ coverage values, the energy barrier for $\mathrm{H}$ chemisorption on $\mathrm{NiP}$ appears to increase by more than double than the original value. It is thought that the higher $\mathrm{H}$-coverage on NiP inhibits further absorption of $\mathrm{H}$, resulting in the overall lowering of the HER efficiency. We further consider the contribution of various transition metal dopants (Fe, Co, Mn, and Mo) at 1/4 and then 1/8 ML H coverages (Figures $10 \mathrm{~b}$ and 10c). The doping that we modelled was based on the substitution of $\mathrm{Ni}$ by $\mathrm{M}(\mathrm{M}=\mathrm{Fe}$, $\mathrm{Co}, \mathrm{Mn}$, and Mo). As seen from the two figures, their $\Delta G$ values are highly dependent on the degree of $\mathrm{H}$ coverage.[19,38] As previously discussed on the 'volcano relationship' by Nørskov and co-workers,[19] the $\Delta G$ value closer to the thermoneutral line $(0 \mathrm{eV})$ whereby the thermodynamic $\mathrm{H}^{+}$reduction to $\mathrm{H}_{2}$ production is reached, gives higher HER efficiency. Since larger positive $\Delta G$ value represents too weak $H$ adsorption (disfavour for Volmer step but favour for Tafel step) while larger negative $\Delta G$ denotes too strong H-adsorption (favour for Volmer step but disfavour for Tafel step), the $\Delta G$ value near $0 \mathrm{eV}$ therefore suggests the optimal HER performance.

In a higher $\mathrm{H}$ coverage (Figure 10b) on the NiMP surface, the $\mathrm{H}$ absorption energy profile on NiCoP surface clearly gives the best HER performance as it gives only $-0.03 \mathrm{eV}$. This can explain the fact that the use of water with higher proton concentration (eosin Y) renders this catalyst the most active for HER compared to the other (see Figure 9). However, the NiFeP surface does not favour for $\mathrm{H}^{+}$adsorption and reduction while both NiMoP and $\mathrm{NiMnP}$ systems are energetically unfavourable for HER. In contrast, in a lower H coverage (Figure 10c), as our case using ruthenium complex in DMF, both NiMoP and NiMnP become energetically favourable for higher HER activity, whereas for the NiCoP system, the $\Delta G$ is far from zero value. Under this situation, the $\mathrm{H}$ desorption may be inhibited due to too strong $\mathrm{M}$ $\mathrm{H}$ bonding. 
Figure 10d illustrates the changes of free energy with $\mathrm{H}$ coverage for pristine $\mathrm{NiP}$ and NiMP surfaces, with respect to the thermoneutral line. Both Co and Fe insertion improve their HER activities at higher $\mathrm{H}$ coverage, while in the case of $\mathrm{Fe}$, it varies much more gently with the $\mathrm{H}$ coverage. This indicates that NiFeP gives good HER performance only under an extremely high $\mathrm{H}$ coverage. But $\mathrm{NiCoP}$ is much sensitive to the $\mathrm{H}$ coverage value. It is noteworthy that their relative HER performance changes depending on the H-coverage. On the other hand, the pristine NiP, NiMnP and NiMoP surfaces all exhibit positive slope at increasing $\mathrm{H}$ coverage, which would lower the HER activities.

Based on these DFT calculations, we conclude that NiMoP gives superior HER rate at the low $\mathrm{H}$ coverage. This is consistent with our observations that this catalyst surface gives highest HER performance in electrochemical and photocatalytic activation at low $\mathrm{H}$ coverage. On the other hand, NiCoP gives the highest HER photocatalytic activity at high $\mathrm{H}$ coverage when aqueous eosin $\mathrm{Y}$ photosensitizer is used. Thus, we demonstrate the highly tunable activities of these single-phase composites dependent on the electron filling of their metallic bands and H-coverage. We also compared the total energy in a physicochemical trend (Figure 10e). It is found that the NiCoP gives more stable configuration than the other NiMP systems.

\section{Conclusions}

In this work, we show that various transition metal dopers, including cobalt, iron, manganese, and molybdenum are successfully added into the nickel phosphide crystal lattice as a single phase without significant structural change. The EXAFS data indicates that the typical Fe gives the same neighboring local bonding with $\mathrm{Ni}$ and $\mathrm{P}$, implying the successful substitution of $\mathrm{Ni}$ in nanoparticle level. The as-synthesized metal-doped nickel phosphide nanoparticles are semi-monodispersed spherical in shape and possessing the same hexagonal crystal structure as $\mathrm{Ni}_{2} \mathrm{P}$ at this high level of doping. The metal-doped and pristine nickel phosphide nanoparticles are shown to maintain the same metallic properties as $\mathrm{Ni}_{2} \mathrm{P}$, which is crucially important as electrode material without introducing impedance to the system. Also, in this study, the rational and tunable properties of nickel phosphide nanoparticles substituted with various metal dopants in both electro- and photochemical HER are clearly demonstrated. For example, Ni $\left(d^{8}\right)$ contains more than sufficient electrons in its $d$-band which leads to a poor HER activity. The rational substitution of Ni by transition metals of lower numbers of $d$ electrons could render the doped nickel phosphide nanoparticles more active than that of pristine nickel phosphide nanoparticle due to the optimal electron filling. Similarly, the extent of Ni substitution by Co can also allow the fine tuning of the electron filling to the metallic bands of NiCoP. Molybdenum-doped nickel phosphide nanoparticle is found to have the lowest overpotential of $0.4 \mathrm{~V} v s$. RHE and a Tafel slope of $163 \mathrm{mV} \mathrm{dec}^{-1}$ in alkaline medium, which is identified as a promising candidate among the various doped samples for the replacement of Pt based catalysts. Also, metal-doped nickel phosphide nanoparticles show promising activity in the dye-sensitized photocatalytic hydrogen evolution reaction. Again, molybdenum-doped nickel phosphide nanoparticle shows the best activity on hydrogen production with a rate of $268 \mathrm{mmol} \mathrm{h}^{-1} \mathrm{~g}^{-1}$ in the presence of $\left[\mathrm{Ru}(\mathrm{bpy})_{3}\right]\left(\mathrm{ClO}_{4}\right)_{2}$ in DMF. On the other hand, cobalt-doped nickel phosphide nanoparticle exhibits the best hydrogen production rate of $20.4 \mathrm{mmol} \mathrm{h}^{-1} \mathrm{~g}^{-1}$ in eosin Y-sensitized photocatalytic system in water. We 
attribute the difference in HER activity to the different degree of $\mathrm{H}$ coverage especially in the case for $\mathrm{Co}$, which can also be the key factor of influencing the HER activity exerted by the environment.

Overall, it is demonstrated the $\mathrm{Ni}$ sites on $\mathrm{Ni}_{2} \mathrm{P}$ can be substituted by other transition metals at high doping level, to yield the single phase NiMP solid structure, which displays tunable catalytic properties for HER. This class of materials is a promising candidate to replace Pt used in optical cells for hydrogen production.

\section{Supporting Information}

Supporting Information is available or from the author.

\section{Acknowledgements}

We thank B18 of Diamond light source, UK to provide access to XAS analysis of the materials. 


\section{References}

[1] T. S. Teets and D. G. Nocera, Chem. Commun., 2011, 47, 9268.

[2] F. Wang, W. G. Wang, H. Y. Wang, G. Si, C. H. Tung and L. Z. Wu, ACS Catal., 2012, 2, 407.

[3] S. M. Arachchige and K. J. Brewer, Energy Production and Storage: Inorganic Chemical Strategies for a Warming World, John Wiley \& Sons, Chichester, West Sussex, UK, 2010.

[4] C. G. Morales-Guio, L. A. Stern and X. Hu, Chem. Soc. Rev., 2014, 43, 6555.

[5] E. Aharon-Shalom and A. Heller, J. Electrochem. Soc., 1982, 129, 2865.

[6] A. Fujishima and K. Honda, Nature, 1972, 238, 37.

[7] Y. Li, H. Wang, L. Xie, Y. Liang, G. Hong and H. Dai, J. Am. Chem. Soc., 2011, 133, 7296.

[8] P. Liu and J. A. Rodriguez, J. Am. Chem. Soc., 2005, 127, 14871.

[9] E. J. Popczun, J. R. McKone, C. G. Read, A. J. Biacchi, A. M. Wiltrout, N. S. Lewis and R. E. Schaak, J. Am. Chem. Soc., 2013, 135, 9267.

[10] Z. Huang, Z. Chen, Z. Chen, C. Lv, H. Meng and C. Zhang, ACS Nano, 2014, 8, 8121.

[11] M. Ledendecker, S. K. Calderón, C. Papp, H.-P. Steinruck, M. Antonietti and M. Shalom, Angew. Chem., Int. Ed., 2015, 54, 12361.

[12] Y. Pan, Y. Liu, J. Zhao, K. Yang, J. Liang, D. Liu, W. Hu, D. Liu, Y. Liu and C. Liu, J. Mater. Chem. A, 2015, 3, 1656.

[13] Y. Pan, W. H. Hu, D. P. Liu, Y. Q. Liu and C. G. Liu, J. Mater. Chem. A, 2015, 3, 13087.

[14] Y. Bai, H. Zhang, X. Li, L. Liu, H. Xu, H. Qiu and Y. Wang, Nanoscale, 2015, 7, 1446.

[15] A. Han, S. Jin, H. L. Chen, H. X. Ji, Z. J. Sun and P. W. Du, J. Mater. Chem. A, 2015, 3, 1941.

[16] D. Zeng, W. Xu, W.-J. Ong, J. Xu, H. Ren, Y. Chen, H. Zheng and D.-L. Peng, Appl. Catal., B, 2018, 221, 47.

[17] Z. Sun, H. Zheng, J. Li and P. Du, Energy Environ. Sci., 2015, 8, 2668.

[18] S. Cao, Y. Chen, C.-J. Wang, P. He and W.-F. Fu, Chem. Commun., 2014, 50, 10427.

[19] J. K. Nørskov, T. Bligaard, A. Logadottir, J. R. Kitchin, J. G. Chen, S. Pandelov and U. Stimming, J. Electrochem. Soc., 2005, 152, J23.

[20] J. Kibsgaard, C. Tsai, K. Chan, J. D. Benck, J. K. Nørskov, F. Abild-Pedersen and T. F. Jaramillo, Energy Environ. Sci., 2015, 8, 3022.

[21] J. Greeley, T. F. Jaramillo, J. Bonde, I. Chorkendorff and J. K. Nørskov, Nat. Mater., 2006, 5, 909.

[22] Y. Li, P. Hasin and Y. Wu, Adv. Mater., 2010, 22, 1926.

[23] Y. Yang, H. Fei, G. Ruan, C. Xiang and J. M. Tour, ACS Nano, 2014, 8, 9518.

[24] S. Chen and S.-Z. Qiao, ACS Nano, 2013, 7, 10190.

[25] C. Zhang, Y. Xie, H. Deng, C. Zhang, J.-W. Su, Y. Dong and J. Lin, Int. J. Hydrogen Energy, 2018, 43, 7299.

[26] J. Li, M. Yan, X. Zhou, Z.-Q. Huang, Z. Xia, C.-R. Chang, Y. Ma and Y. Qu, Adv. Funct. Mater., 2016, 26, 6785.

[27] Y. Li, J. Liu, C. Chen, X. Zhang and J. Chen, ACS Appl. Mater. Interfaces, 2017, 9, 5982. 
[28] L. Bi, X. Gao, L. Zhang, D. Wang, X. Zou and T. Xie, ChemSusChem, 2018, 11, 276.

[29] F. Lyu, Y. Bai, Q. Wang, L. Wang, X. Zhang and Y. Yin, Dalton Trans, 2017, 46, 10545.

[30] H.-R. Seo, K.-S. Cho, S.-H. Kim and Y.-K. Lee, J. Korean Phys. Soc., 2010, 56, 2083.

[31] M. M. Jaksic, Int. J. Hydrogen Energy, 2001, 26, 559

[32] E. Navarro-Flores, Z. Chong and S. Omanovic, J. Mol. Catal. A: Chem., 2005, 226, 179.

[33] M. M. Jaksic, Int. J. Hydrogen Energy, 2001, 26, 559.

[34] J. Willkomm, K. L. Orchard, A. Reynal, E. Pastor, J. R. Durrant and E. Reisner, Chem. Soc. Rev., 2016, 45, 9.

[35] C. Tsai, K. Chan, J. K. Nørskov and F. Abild-Pedersen, Surf. Sci., 2015, 640, 133.

[36] X. Fan, S. Wang, Y. An and W. Lau, J. Phys. Chem. C, 2016, 120, 1623.

[37] J. Durst, A. Siebel, C. Simon, F. Hasche, J. Herranz and H. A. Gasteiger, Energy Environ. Sci., 2014, 7, 2255.

[38] K. Kalyanasundaram, Coord. Chem. Rev., 1982, 46, 159. 


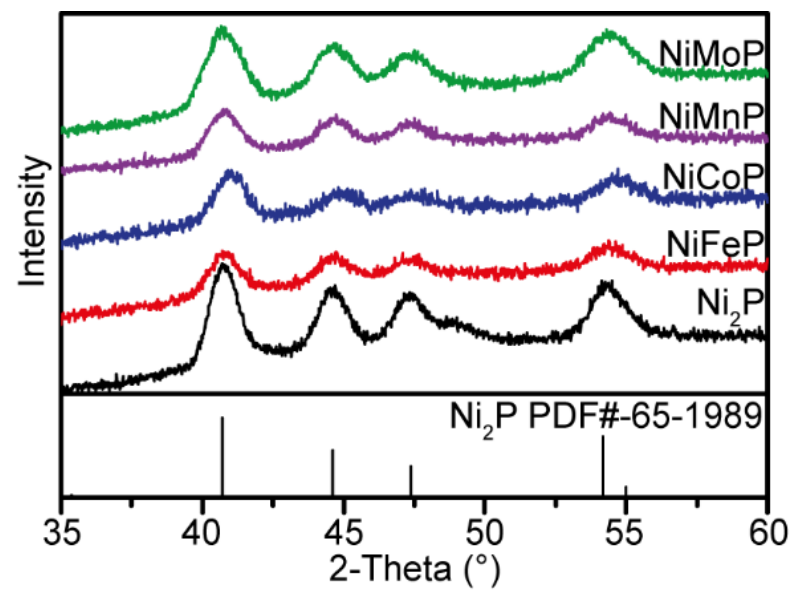

Figure 1. XRD patterns of $\mathrm{Ni}_{2} \mathrm{P}$ and various NiMP nanoparticles.

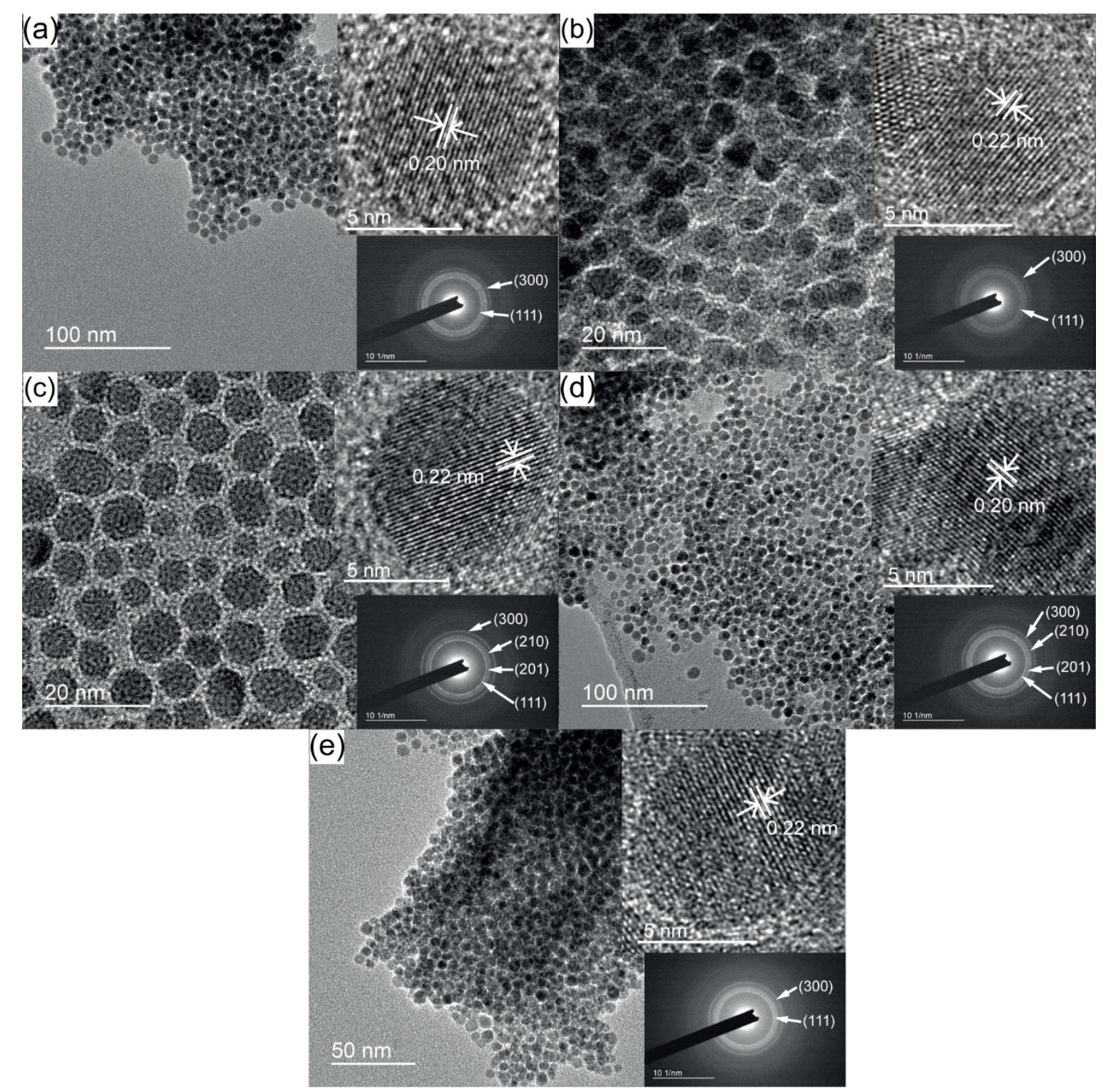

Figure 2. TEM images of inset. (a) $\mathrm{Ni}_{2} \mathrm{P}$, (b) $\mathrm{NiFeP}$, (c) $\mathrm{NiCoP}$, (d) $\mathrm{NiMnP}$, and (e) $\mathrm{NiMoP}$ nanoparticles. HR-TEM images and selected area electron diffraction patterns are shown as inset. 

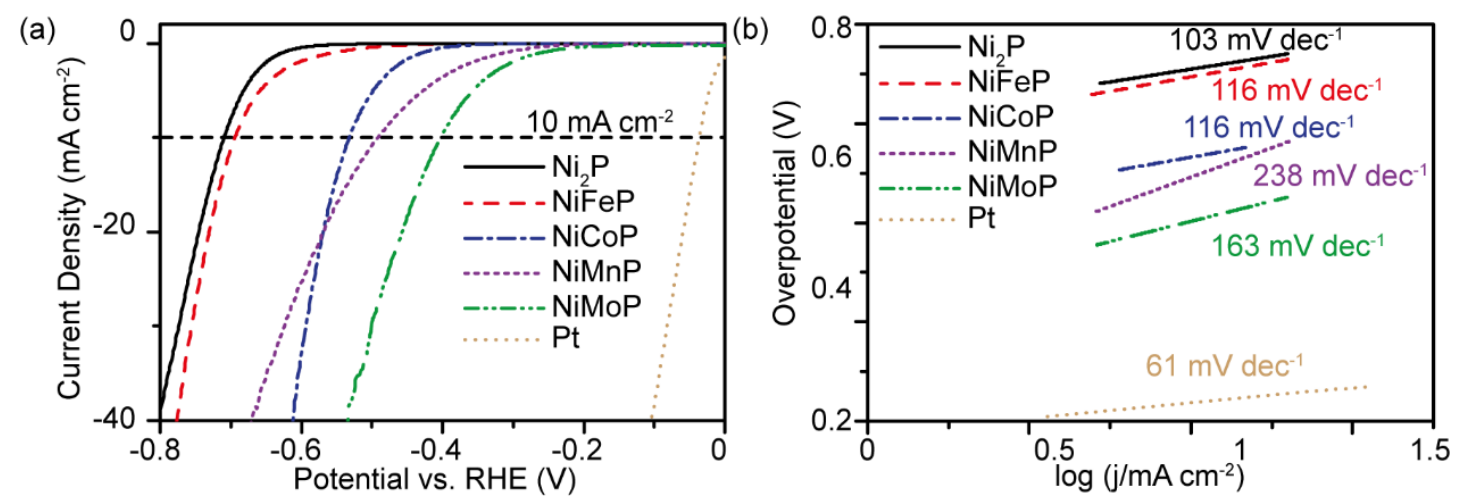

Figure 3. (a) Linear sweep voltammograms and (b) Tafel plots of $\mathrm{Ni}_{2} \mathrm{P}$ and various NiMP nanoparticles in $1 \mathrm{M} \mathrm{KOH}$.

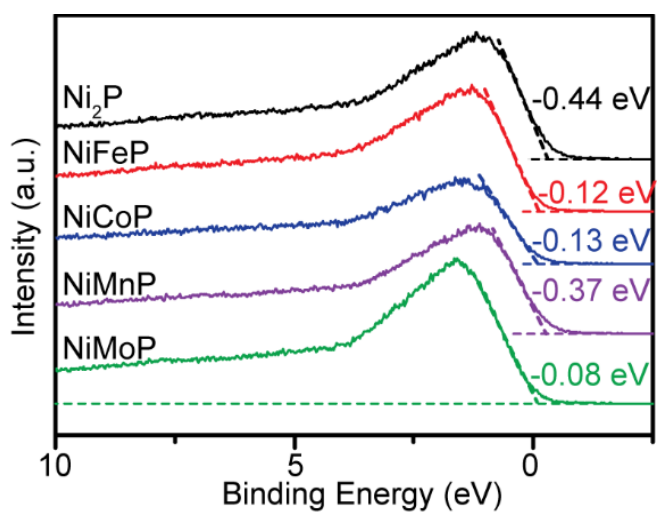

Figure 4. VB-XPS spectra of various NiMP nanoparticles. 

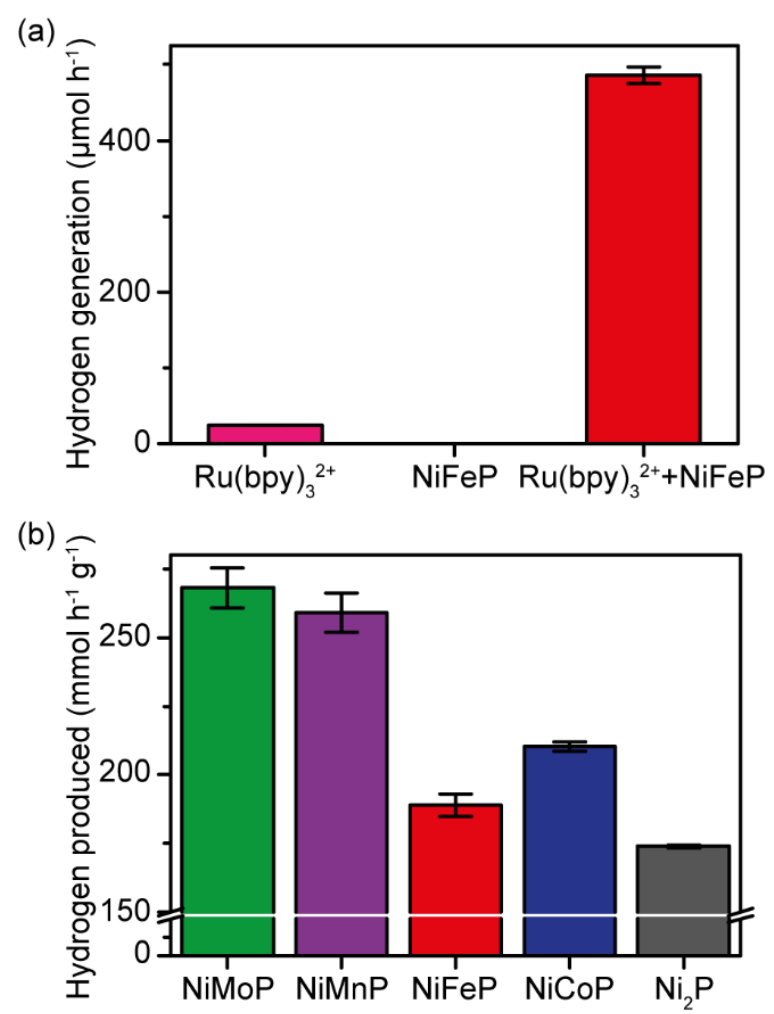

Figure 5. (a) Comparison of the amount of $\mathrm{H}_{2}$ generated on an hourly basis in the presence of $\left[\mathrm{Ru}(\text { bpy })_{3}\right]\left(\mathrm{ClO}_{4}\right)_{2}$ only, NiFeP only, and both $\left[\mathrm{Ru}(\mathrm{bpy})_{3}\right]\left(\mathrm{ClO}_{4}\right)_{2} / \mathrm{NiFeP}$. (b) Photocatalytic activity of pristine and various NiMP nanoparticles are compared on an hourly basis per mass of phosphide-based catalyst. (DMF solvent is used).

(a)

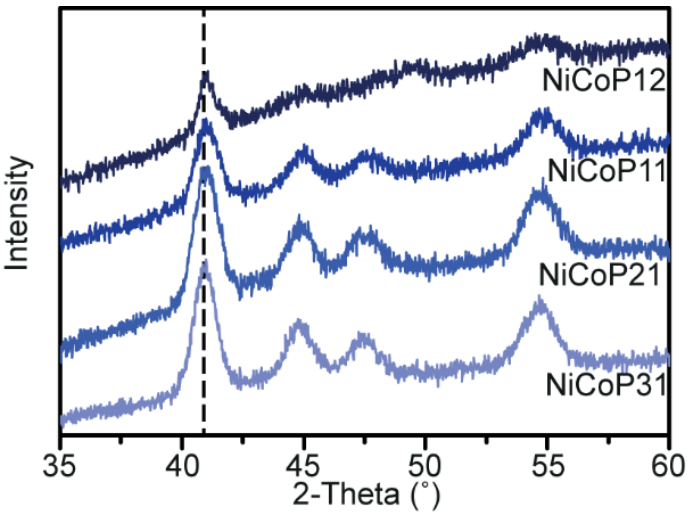

(b)

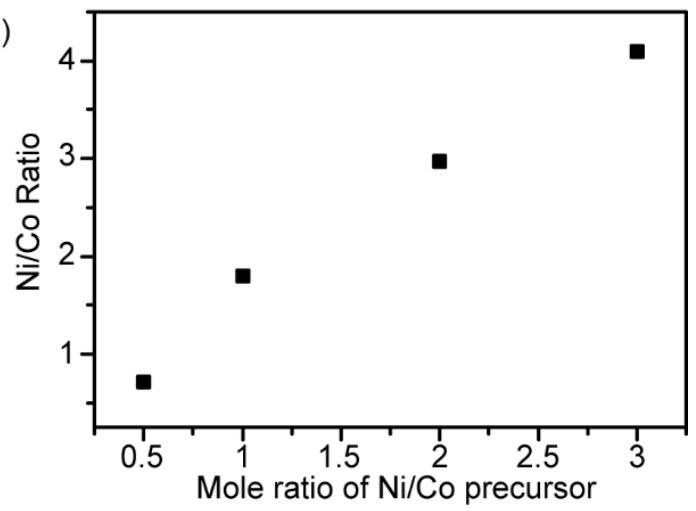

Figure 6. (a) XRD pattern of various NiCoP nanoparticles prepared using different Ni:Co precursor ratios and (b) relationship of elemental ratio between actual and loading nickel-tocobalt ratios. 


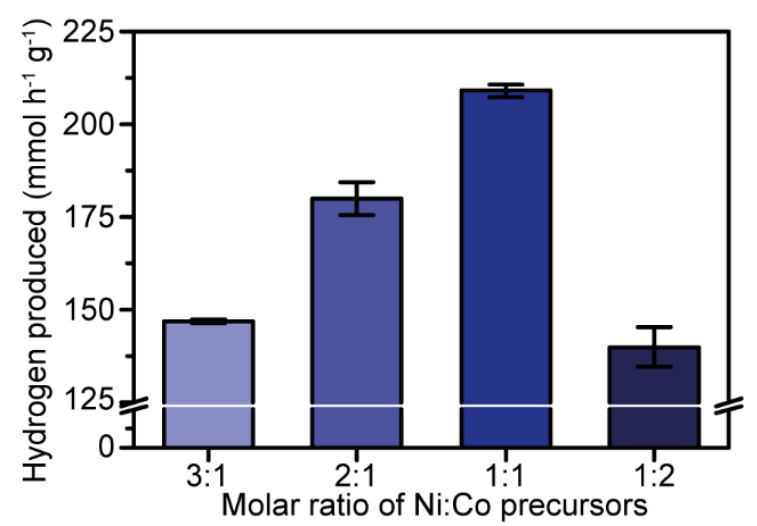

Figure 7. Effect of molar ratio of Ni:Co precursors on hydrogen production.

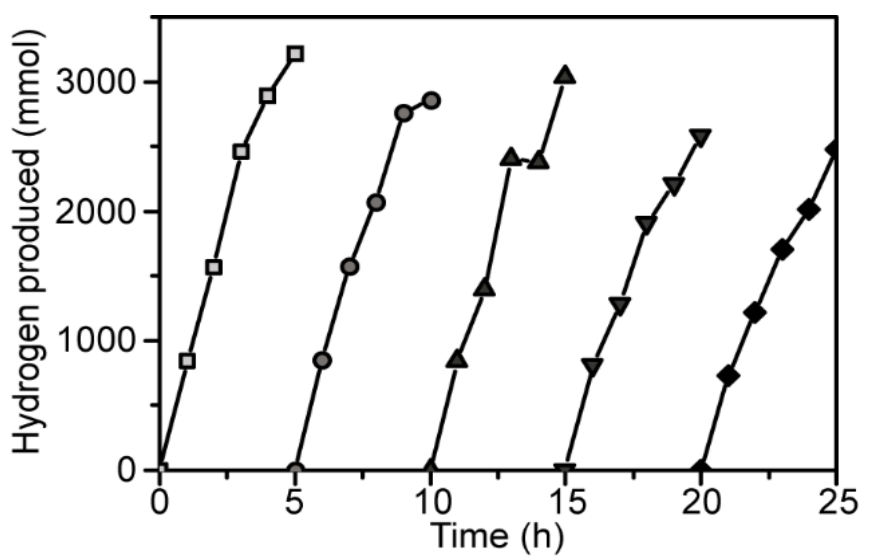

Figure 8. Stability test on NiMoP nanoparticle in $\mathrm{Ru}(\mathrm{bpy}) 3^{2+}$-sensitized photocatalytic HER. Photocatalysis was carried out for $25 \mathrm{~h}$ as 5 separate runs with $\mathrm{Ru}(\mathrm{bpy})_{3}{ }^{2+}$ and sacrificial agent refurnished for each run.

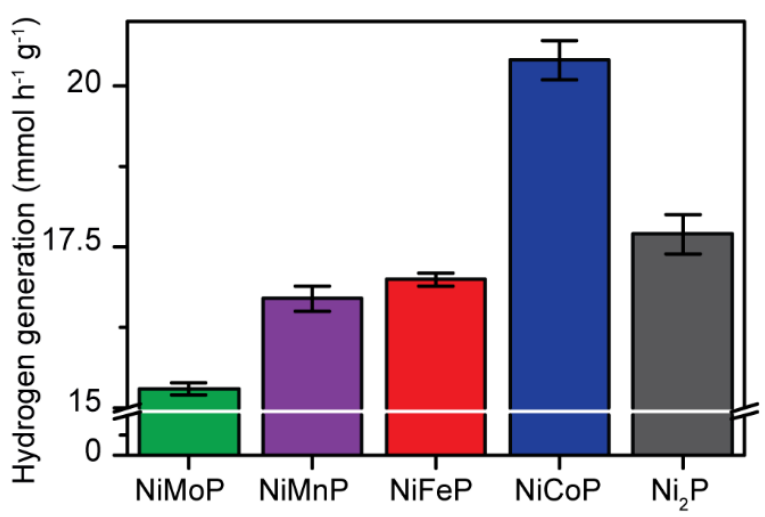

Figure 9. Photocatalytic HER performance of pristine $\mathrm{Ni}_{2} \mathrm{P}$ and various NiMP nanoparticles using eosin $\mathrm{Y}$ as the photosensitizer in $\mathrm{H}_{2} \mathrm{O}$. 

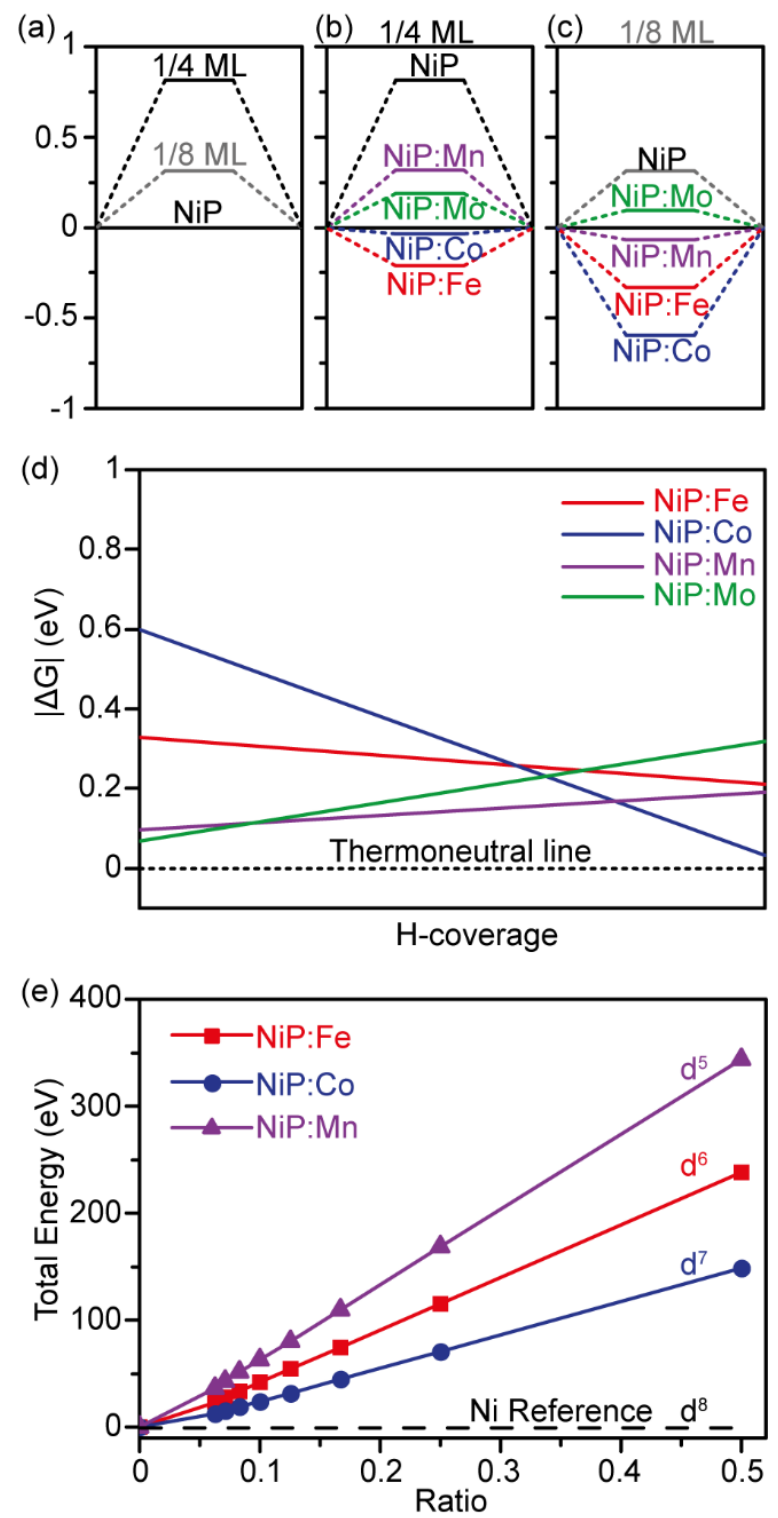

Figure 10. (a) Free energy for $\mathrm{H}$ absorption on pristine NiP with different $\mathrm{H}$ coverage. Comparison of $\Delta G$ for the $\mathrm{H}$ absorption on pristine NiP and NiMP surface systems at the coverage of (b) 1/4 ML and (c) 1/8 ML (M = Fe, Co, Mn, and Mo). (d) Free energy trend compared to the thermoneutral line for pristine NiP and NiMP surface systems. (e) Total energy trend with related to the doping level in NiMP surface systems. 
Table 1. EXAFS of $\mathrm{Ni}_{2} \mathrm{P}$ and $\mathrm{NiFeP}$ nanoparticles.

\begin{tabular}{|c|c|c|c|c|c|c|}
\hline & Bond & Enot* & $\begin{array}{c}\text { Coordination } \\
\text { number }\end{array}$ & SS & $\begin{array}{c}\text { Bond length } \\
(\AA)\end{array}$ & R-factor \\
\hline \multirow{2}{*}{$\mathrm{Ni}_{2} \mathbf{P}$} & Ni-P & \multirow{2}{*}{2.7} & $2.9(2)$ & $0.008(1)$ & $2.25(1)$ & \multirow{2}{*}{$0.6 \%$} \\
\hline & $\mathrm{Ni}-\mathrm{Ni}$ & & $3.3(3)$ & $0.008(1)$ & $2.59(1)$ & \\
\hline \multirow{4}{*}{ NiFeP } & Ni-P & \multirow{2}{*}{4.3} & $2.3(3)$ & $0.008(2)$ & $2.24(1)$ & \multirow{2}{*}{$1.3 \%$} \\
\hline & $\mathrm{Ni}-\mathrm{Ni}$ & & $2.5(3)$ & $0.006(1)$ & $2.59(1)$ & \\
\hline & Fe-P & \multirow{2}{*}{5.5} & $3.7(3)$ & $0.007(1)$ & $2.21(1)$ & \multirow{2}{*}{$2.0 \%$} \\
\hline & $\mathrm{Fe}-\mathrm{Ni}$ & & $3.6(3)$ & $0.005(1)$ & $2.63(1)$ & \\
\hline
\end{tabular}

* Enot is the energy difference between the absorption energies in experimental and calculated values.

Table 2. Summary of electrochemical parameters of $\mathrm{Ni}_{2} \mathrm{P}$ and various NiMP nanoparticles.

\begin{tabular}{|c|c|c|c|}
\hline & $\begin{array}{l}\text { Overpotential at } \\
10 \mathrm{~mA} \mathrm{~cm} \text { (V) }\end{array}$ & Tafel Slope (mV dec $\left.{ }^{-1}\right)$ & $\begin{array}{l}\text { Exchange Current } \\
\text { Density }(\mathrm{mA} \mathrm{cm})\end{array}$ \\
\hline NiMoP & -0.4 & 163 & 0.03 \\
\hline NiMnP & -0.49 & 238 & 0.08 \\
\hline NiFeP & -0.69 & 116 & $1 \times 10^{-5}$ \\
\hline NiCoP & -0.53 & 116 & $2.5 \times 10^{-4}$ \\
\hline $\mathrm{Ni}_{2} \mathbf{P}$ & -0.71 & 103 & $1.3 \times 10^{-6}$ \\
\hline
\end{tabular}

